\title{
The Variation of Galaxy Morphological Type with the Shear of Environment
}

\author{
Jounghun Lee and Bomee Lee \\ Department of Physics and Astronomy, FPRD, Seoul National University, Seoul 151-747, \\ Korea \\ jounghun@astro.snu.ac.kr, bmlee@astro.snu.ac.kr
}

\begin{abstract}
Recent N-body simulations have shown that the assembly history of galactic halos depend on the density of large-scale environment. It implies that the galaxy properties like age and size of bulge may also vary with the surrounding large-scale structures, which are characterized by the tidal shear as well as the density. By using a sample of 15,882 well-resolved nearby galaxies from the Tully Catalog and the real space tidal field reconstructed from the 2Mass Redshift Survey (2MRS), we investigate the dependence of galaxy morphological type on the shear of large-scale environment where the galaxies are embedded. We first calculate the large scale dimensionless overdensities $(\delta)$ and the large-scale ellipticities $(e)$ of the regions where the Tully galaxies are located and classify the Tully galaxies according to their morphological types and create subsamples selected at similar value of $\delta$ but span different ranges in $e$. We calculate the mean ellipticity, $\langle e\rangle$, averaged over each subsample and find a signal of variation of $\langle e\rangle$ with galaxy morphological type: For the case of $0.5 \leq \delta \leq 1.0$, the ellipticals are found to be preferentially located in the regions with low ellipticity. For the case of $-0.3 \leq \delta \leq 0.1$, the latest-type spirals are found to be preferentially located in the regions with high ellipticity. The null hypothesis that the mean ellipticities of the regions where the ellipticals and the latest type spirals are located are same as the global mean ellipticity averaged over all types is rejected at $3 \sigma$ level when $-0.3 \leq \delta \leq 0.1$. Yet, no signal of galaxy-shear correlation is found in the highly overdense/underdense regions. The observed trend suggests that the formation epochs of galactic halos might be a function not only of halo mass and large-scale density but also of large-scale shear. Since the statistical significance of the overall trend is low, it will require a sample of at least 100, 000 galaxies to verify the existence of this correlation.
\end{abstract}

Subject headings: cosmology:theory — galaxies: observation 


\section{INTRODUCTION}

It has been known for long that the physical properties of galaxies such as morphological type, color, luminosity, spin parameter, star formation rate, concentration parameter and so on are functions of their environments (Dressler 1980; Postman \& Geller 1984; Whitmore et al. 1993; Lewis et al. 2002; Gomez et al. 2003; Goto et al. 2003; Roias et al. 2005; Kuehn \& Ryden 2005; Blaton et al. 2005; Bernardi et al. 2006; Choi et al. 2007; Park et al. 2007). Most previous works have largely focused on the galaxies located in the highly dense regions and usually quantified the environmental dependence of galaxy properties in terms of cross-correlations with local density on small scale, which is believed to be established by environment-dependent processes like galaxy-galaxy interaction.

The currently popular $\Lambda \mathrm{CDM}$ model predicts that the galaxy properties are correlated with not only the small scale but also the large-scale environment. Here the small scale and the large-scale environments represent the surrounding regions smaller and larger than $5 h^{-1} \mathrm{Mpc}$, respectively. Recent high-resolution N-body simulations of a $\Lambda$ CDM cosmology have demonstrated that the assembly history of galactic halos of a given mass change with the density field smoothed on sufficiently large scale (Gao et al. 2005). Since the galaxy content depend strongly on assembly history of its host halo (Springel et al. 2005; Croton et al. 2007), this numerical finding suggests that the intrinsic properties of galaxies may be correlated with the density of large-scale environments.

In fact, several groups have already found observational evidences for the existence of cross-correlations between galaxy properties and large-scale tidal shear. Navarro et. al. (2004) found that the spin axes of spiral galaxies near the Local Supercluster lie preferentially on the Supergalactic Plane. Trujillo et. al. (2006) also showed by analyzing recent observational data from large galaxy surveys that the spin axes of void galaxies tend to be inclined to the void surfaces, keeping the initial memory of tidally induce alignments. Pandey \& Bharadwaj (2006) have shown that the galaxy luminosity and colour are dependent on the filamentarity of large-scale environment. More recently, Pandey \& Bharadwaj (2008) have also presented that the star formation rate of galaxies are cross-correlated with the filamentarity of the surrounding large-scale structures. Hernandez et al. (2007) found a one-to-one correspondence between the value of the galaxy spin parameter and the morpho-

logical type, while Hernandez \& Cervantes-Sodi (2006) and Cervantes-Sodi \& Hernandez (2008) have noted that the spin parameter of the observed galaxies is strongly correlated with their color and chemical abundance.

The existence of cross-correlations between galaxy properties and density of large-scale environment implies that the galaxy content still have the memory of the initial conditions of the Lagrangian regions where the host halos formed. In the scenario of the biased galaxy 
formation, the galaxy sites correspond to the high peaks of the linear density field (Kaiser 1984; Bardeen et al. 1986). But, since the initial density peaks are not spherical, they should be characterized not only by the peak heights but also by their ellipticity and prolateness. Hence, if the galaxy properties are still linked to the large-scale density, then it is likely that they are also linked to the large-scale ellipticity and prolaticity.

Our goal here is to test this statistical link. Instead of the ellipticity of the large-scale density field, however, we consider the ellipticity of the large-scale potential field (i.e., the tidal shear). Since the potential field is smoother than the density field, we believe that the large-scale potential field reflects more directly the linear conditions. Furthermore, the tidal shear field, defined as the second derivative of the potential field, has a large-scale coherence, resulting in highly anisotropic spatial distribution of galaxies at present epoch (Bond et al. 1996). Therefore, the tidal shear field is more readily measurable from the large-scale spatial distribution of present galaxies.

In previous approaches, however, the correlation between galaxy properties and the shear of large-scale structure was measured in redshift space. Since the redshift distortion effect could contaminate the measurements of the filamentarity of the large scale structure, it will be desirable to measure it in real space. Moreover, true as it is that in the linear regime the density and the tidal shear at a given region are mutually independent, it is likely that the two quantities have developed cross-correlations in the subsequent non-linear regime. Therefore, to find an independent relationship between galaxy properties and large-scale tidal shear, one has to first account for the density-shear correlations and remove its effect.

We attempt here to measure observationally an independent relationship between the morphological types of nearby large galaxies and the real-space tidal shear reconstructed from all sky survey. The organization of this paper is as follows: In $\S 2$, the observational data are described. In $\S 3$, the shear of environment is defined and its mean value averaged over each galaxy subsample at similar density is measured as a function of galaxy morphological type. In $\S 4$, the results are discussed and a final conclusion is drawn.

\section{OBSERVATIONAL DATA: AN OVERVIEW}

A total of 35, 000 galaxies with spectroscopic information are listed in the Tully Catalog, which was constructed from the ESO/Uppsala full-sky survey (Nilson 1974; Lauberts 1982). Only 15, 922 galaxies in the Tully catalog have detailed information on their morphological types, which were determined by B. Tully according to the conventional Hubble classification scheme. For a detailed description of the Hubble classification scheme, see Table 2 in the 
Third References Catalog of Bright Galaxies (RC3 de Vaucouleurs et al. 1991). As for the rest 19, 078 galaxies in the Tully catalog, the stages of their morphological types are described as either "irregulars" or "uncertain" or "doubtful" or "spindle", "outer ring" or "pseudoouter R", or "irregulars". These are thus excluded from our analysis.

According to the morphological type described in RC3, we divide the selected Tully galaxies into six samples: E, L, SI, SII, SIII, and SIV. Table1lists the class, the morphological type (as in RC3), and the number of the galaxies $\left(N_{g}\right)$ belonging to the six samples: The sample "E" contains only ellipticals with the Hubble type of cE,E0, E0-1, E+; The sample $L$ contains only lenticulars with the Hubble type of $S 0^{-1}, S 0^{O}, S 0^{+}$; The sample $S I$ consists of the spirals of types $S 0 / a$ and $S a$ which have tightly wound arms and a largest bulge. While the spirals of types $S c d, S d, S d m$ and $S m$ belong to the sample $S I V$, which have no well developed bulge and loosely wound arms. The sample $S I I$ contains the spirals of type $S a$ as well as the spirals of type $S a b$ that are intermediate between $S a$ and $S b$. Likewise, the sample $S I I I$ contains the spirals type of $S b$ and the spirals of type $S b c$. As can be noted, the size of bulge and the tightness of the spiral arms decrease from $S I$ to $S I V$.

The real space density field was originally reconstructed on $64^{3}$ grids in a box of linear size $400 h^{-1} \mathrm{Mpc}$ from the 2Mass Redshift Survey (Erdogdu et al. 2006, 2MRS). Basically, it is the linear density field smoothed with a wiener filter assuming a linear bias. Lee \& Erdogdu (2007) used the $2 \mathrm{MRS}$ density field to reconstruct the tidal shear field on the same $64^{3}$ grids. As described in detail in Erdogdu et al. (2006), the real space positions were recovered by applying the Wiener reconstruction algorithm to the $2 \mathrm{MRS}$ data. This algorithm basically deconvolve the linear redshift distortions in the radial direction using a distortion matrix. For a detailed description of the method used to recover the real-space position, see Erdogdu et al. (2006).

The reconstructed tidal shear field consists of a set of three eigenvalues, $\left\{\lambda_{1}, \lambda_{2}, \lambda_{3}\right\}$, of the tidal shear tensor assigned to each grid. Figure 1 plots the probability density distributions of the three eigenvalues of the $2 \mathrm{MRS}$ tidal field, assuming $\lambda_{1} \geq \lambda_{2} \geq \lambda_{3}$. As can be seen, the distribution of the second largest eigenvalue, $p\left(\lambda_{2}\right)$, is almost symmetric around the peak at zero with narrow width, while the other two distributions $p\left(\lambda_{1}\right)$ and $p\left(\lambda_{3}\right)$ are asymmetric around zero with broader shapes.

The differences among the eigenvalues are related to the shear of environment at a given grid. If $\lambda_{1}=\lambda_{2}=\lambda_{3}$, it means that the shear of environment is zero. The larger the mutual differences among the eigenvalues are, the higher the shear of environment is. Figure 2 plots the 2MRS tidal shear eigenvalue field evaluated on a thin shell at $80 h^{-1} \mathrm{Mpc}$ shown in Supergalactic Aitoff projection. The top, middle and bottom panel shows the $\lambda_{1}, \lambda_{2}$ and $\lambda_{3}$ field, respectively. In each panel, the darker regions correspond to the low eigenvalues while 
the bright regions to the high eigenvalues.

By applying the cloud-in-cell (CIC) interpolation method (Hockney \& Eastwood 1988) to the 2MRS tidal shear field, Lee \& Erdogdu (2007) also calculated the tidal tensors and their eigenvalues at the positions of the selected 15,922 Tully galaxies. Using the reconstructed 2MRS tidal shear field, we measure the values of the shear at the positions of the Tully galaxies. In $\S 3$, we will see how this measured shears are related to the morphological types of the Tully galaxies.

\section{Galaxy-Shear Correlations}

\section{1. $\quad$ Variation with the LSS Type}

Let us consider a position, $\mathbf{x}$, where a selected Tully galaxy is located. It depends on the signs of $\lambda_{1}, \lambda_{2}, \lambda_{3}$ at $\mathbf{x}$, whether the selected galaxy belongs to a void or a sheet or a filament or a halo. If all three eigenvalues are positive at $\mathbf{x}$, then the given galaxy is located in a halo-like region; If $\lambda_{2}>0, \lambda_{3}<0$, then it is in a filament-like region; If $\lambda_{1}>0, \lambda_{2}<0$, then it is in a sheet-like region; If $\lambda_{1}<0$, then it is in a void-like region.

We investigate the dependence of the relative abundance of the sample galaxies located in halo-like regions on the morphological type, by measuring the conditional number density (CND):

$$
\frac{\delta N_{\text {type }}}{\delta N_{\text {all }}} \equiv \frac{\Delta N_{\text {type }}}{N_{\text {type }}} \times \frac{N_{\text {all }}}{\Delta N_{\text {all }}} .
$$

Here, $N_{\text {type }}$ is the number of the galaxies belonging to a given sample, $\Delta N_{\text {type }}$ is the number of those galaxies in the sample which are located in halo-like regions, $N_{\text {all }}$ is the number of all selected Tully galaxies and $\Delta N_{\text {all }}$ is the number of those Tully galaxies which are located in halo-like regions. If CND $>1$, then the galaxies in a given sample have stronger tendency to be located in halo-like regions than the parent sample.

The CND of the galaxies located in void-like, sheet-like, filament-like regions are also calculated in a similar manner. Figure 3 plots the CNDs as histograms for the six samples with Poisson errors. As can be seen, the six histograms show all distinct behaviors: The CND of the sample $\mathrm{E}$ is highest in halo-like regions and lowest in void-like regions; The CND of the sample L is lowest in sheet-like regions; The CND of the sample SI is highest in filament-like regions; The CND of the sample SII is almost uniform; The CND of the sample SIII is highest in void-like and sheet-like regions; The CND of the sample SIV is highest in void-like regions. It is interesting to see that in void-like regions the CND of the lenticulars have relatively high value compared with that of the ellipticals. 
Although the result shown in Fig. 3 shows clearly that the galaxy morphology is correlated with the shear of the surrounding large-scale structure, it should not be directly translated into an observational evidence for the existence of an independent relationship between galaxy morphology and large-scale shear. Since the signs of the three eigenvalues of the tidal shears are correlated with the local density, the observed signals shown in Fig. 3 could be resulted from the well known correlations between the local density and the galaxy morphology. For instance, the observed tendency that the ellipticals are more abundant in the halo-like regions may be due to the fact that the local density is usually higher in the halo-like regions since the three eigenvalues are all positive and the ellipticals are preferentially located in the high-density regions. Therefore, to detect the true relationship between galaxy morphology and the shear of large-scale structure, one has to use controlled subsamples where the correlations between galaxy morphology and density are removed. In the following subsection, we pursue this task.

\section{2. $\quad$ Variation with the Ellipticity}

The shear of environment at a given galaxy position $\mathbf{x}$ is caused by the anisotropy in the distribution of the surrounding large scale structure which in turn induces asphericity in the gravitational potential $\Phi(\mathbf{x})$. The asphericity of $\Phi(\mathbf{x})$ can be quantified in terms of ellipticity, e, defined as (Bardeen et al. 1986)

$$
e \equiv \frac{\lambda_{1}-\lambda_{3}}{2|\delta|} .
$$

Here the sum of the three eigenvalues of the tidal shear equals the dimensionless overdensity, $\delta \equiv(\rho-\bar{\rho}) / \bar{\rho}$ where $\bar{\rho}$ is the background density: $\delta=\sum_{i=1}^{3} \lambda_{i}$. If the three eigenvalues, $\lambda_{1}, \lambda_{2}, \lambda_{3}$, at $\mathbf{x}$ have the same value, the iso-potential surface at $\mathbf{x}$ has a spherical shape with $e=0$. The larger the differences between $\lambda_{1}$ and $\lambda_{3}$ are, the higher the value of $e$ is. If the spatial distribution of galaxies located in a region is highly anisotropic, then the value of $e$ of the region will be high. In other words, the region has a high ellipticity. The higher the degree of the anisotropy in the spatial distribution of the galaxies in a region increase, the higher value of the ellipticity the region has.

At the position of each Tully galaxy, we determine the values of $\delta$ and $e$. We first calculate the means of $\Delta \delta \equiv \delta-\bar{\delta}_{G}$ averaged separately over each sample listed in Table 1. where $\bar{\delta}_{G}$ is the global mean value of the dimensionless overdensity averaged over all selected Tully galaxies. If $\Delta \delta>0$, there is a tendency of the sample galaxies to be located in high-density regions. If $\Delta \delta<0$, the sample galaxies tend to be located in low-density regions. If $\Delta \delta=0$ for all six samples, there is no correlation between galaxy morphology and 
large-scale density. Figure 4 plots $\langle\Delta \delta\rangle$ of the six samples. As can be seen, there is indeed a strong dependence of galaxy-morphology on the large-scale density, which is consistent with numerical results (Gao et al. 2005).

Now, we calculate the means of $\Delta e \equiv e-\bar{e}_{G}$ averaged separately over each sample, under the constraint that the value of $\delta$ is fixed in some narrow range to remove the effect of morphology-density correlations. Note that $\bar{e}_{G}$ is averaged in the same constrained range of $\delta$. If $\Delta e>0$, there is a independent tendency of the sample galaxies to be located in the high-shear regions. If $\Delta e<0$, the sample galaxies tend to be located in the low-shear regions. If $\Delta e=0$ for all six samples, there is no correlation between galaxy morphology and large-scale shear.

In Fig. 5 we plot $\langle\Delta e\rangle$ of the six samples in the top panel for the case where the value of $\delta$ is fixed in the range of $[-0.3,-0.1]$. The errors, $\sigma$, are calculated as one standard deviation in the measurement of the mean value: $\sigma \equiv \sqrt{\left[\left\langle(\Delta e)^{2}\right\rangle-\langle\Delta e\rangle^{2}\right] /\left(N_{g}-1\right)}$ where $N_{g}$ is the number of the sample galaxies selected at the given density range. To demonstrate that the correlation between morphology and density is effectively removed by constraining the value of $\delta$ to this narrow range of $[-0.3,-0.1]$, we also plot $\langle\Delta \delta\rangle$ of the six samples in the bottom panel. As can be seen, the value of $\langle\Delta \delta\rangle$, is almost uniform over the six subsamples, indicating that the morphology-density correlations are controlled to a negligible level with this constraint of $-0.3 \leq \delta \leq-0.1$. We detect a $2.9 \sigma$ signal that the mean ellipticity of the sample $S I V$ deviates from the global mean ellipticity. This result suggests that the latest-type spirals tend to be located in the regions of high-ellipticity.

Fig. 6 plots the same as Fig. 5 but with a different constraint of $0.05 \leq \delta \leq 0.1$. A $3 \sigma$ signal of morphology-shear correlation is also detected for the sample $S I V$ galaxies. It is consistent with the previous result that the latest-type spirals tend to be preferentially located in the high-shear environment. Fig. 7 plots the same as Fig. 5 but with $\delta$ in the range of $[0.2,0.4]$. We detect a $2.2 \sigma$ signal that the mean ellipticity of the regions where the galaxies of the sample $E$ are located is lower than the global mean ellipticity, and a $2.4 \sigma$ signal that the mean ellipticity of the regions where the galaxies of the sample $S I V$ is located is higher than the global mean ellipticity. Fig. 8 plots the same as Fig. 5 but with $\delta$ in the range of $[0.5,1.06]$. There are detected a $3.1 \sigma$ signal that the mean ellipticity of the regions where the sample $E$ galaxies are located is lower than the global mean ellipticity, and a $2 \sigma$ signal of correlation between latest type spirals (SIV) and high-shear region. The results shown in Figs. 7 and 8 indicate consistently that the ellipticals tend to be preferentially located in the low-shear environment while the latest-type spirals tend to be preferentially located in the high-shear environment.

Nevertheless, it has to be noted that the galaxy-shear correlation signal is found to be 
statistically significant only in bins containing E and SIV galaxies. Therefore, when the results over all six bins are considered the null hypothesis of no galaxy-shear correlation is still quite acceptable. In the given density range, the null hypothesis is found to be rejected at only the $\sim 10 \%$ confidence level when the results over all six bins are considered. Since we consider only those galaxies at similar densities, the sample size, $N_{g}$, is quite small, resulting in large statistical errors. It is also worth mentioning that neither in the highly underdense region with $\delta<-0.5$ nor in the highly overdense region with $\delta>1.0$, no signal of galaxyshear correlation is found since the degree of density-shear correlations is too high to be removed in these regions.

\section{DISCUSSION AND CONCLUSION}

By measuring observationally the mean ellipticities of large-scale structures where the nearby galaxies are embedded as a function of galaxy morphological types, we have tentatively detected an independent relationship between galaxy morphology and shear of environment: In the mildly overdense environment with large scale dimensionless overdensity of $0.05 \leq \delta \leq 1.06$, the ellipticals are found to prefer the low-shear region; In the mildly underdense environment with large scale density of $-0.3 \leq \delta \leq-0.1$, the latest-type spirals (Scd-Sm) are found to prefer the high-shear region. No correlation signal, however, is found either in the highly overdense $(\delta>1)$ or in the highly underdense environment $(\delta<-0.5)$.

This observational results may be explained by the dependence of halo formation epochs on the shear of large-scale environment. It has been known that the formation epochs of galactic halos depend not only on their mass but also on the density of large-scale environment (Gao et al. 2005). Here we argue that there is some observational evidence to support the hypothesis that the formation epochs of halos also depend on the shear of the largescale environment. In the high-shear environment the halos formed relatively early without growing at late time due to strong tidal disruption from the surrounding matter distribution. Thus, the late-type spirals are likely to be located in this high-shear environment. Meanwhile in the low-shear environment the halos formed relatively recently since there is no strong tidal distribution, having grown at late times through hierarchical merging. Hence, the ellipticals are likely to be found in this low-shear environment. In the highly overdense regions, however, even in case that there is tidal disruption from the surrounding matter distribution, the other stronger effects of environmental processes should mask the shear-dependence of galaxy properties. Likewise in the highly underdense regions, the tidal field is too weak to produce any significant galaxy-shear correlation. It will be interesting to test against N-body simulations whether or not the formation epochs of galactic halos with similar mass 
at similar density is a function of the ellipticity of large-scale dark matter distribution. Our future work is in this direction.

It should be noted here that our results suffer from low statistical significance due to the small sample size. We have seen only a marginal effect of the large-scale shear for the latest-type spirals (Scd-Sm). Meanwhile no signal has been found for the Sb-Sc galaxies even though both types of galaxies occupy similar regions. To remove the effect of the stronger galaxy-density correlations, we had to constrain the overdensities of galactic regions, which resulted in large errors. The null hypothesis that there is no galaxy-shear correlation over the six bins is found to be rejected at only the $10 \%$ level due to large errors. Given our result, it will require a sample of more than 100,000 galaxies to test the hypothesis at $>90 \%$ confidence level. This line of investigation will provide new insight into the formation and evolution of galaxies in a filamentary cosmic web.

We are very grateful to P. Erdogdu for providing us the $2 \mathrm{MRS}$ density field. We also thank an anonymous referee who helped us improve significantly the original manuscript by making many helpful suggestions. This work is supported by the Korea Science and Engineering Foundation (KOSEF) grant funded by the Korean Government (MOST, NO. R01-2007-000-10246-0).

The 2MRS density field was constructed by Erdogdu et al. (2006) by making use of data products from the Two Micron All Sky Survey, which is a joint project of the University of Massachusetts and the Infrared Processing and Analysis Center/California Institute of Technology, funded by the National Aeronautics and Space Administration and the national Science Foundation, and the NASA/IPAC Extragalactic Database (NED) which is operated by the Jet Propulsion Laboratory, California Institute of Technology, under contract with

the National Aeronautics and Space Administration and the SIMBAD database, operated at CDS, Strasbourg, France.

\section{REFERENCES}

Bardeen, J.M., Bond, J.R.,Kaiser, N., \& Szalay, A.S. 1986, ApJ, 304, 15

Bernardi, M., Nichol, R. C., Sheth, R. K., Miller, C. J., \& Brinkmann, J. 2006, ApJ, 131, 1288

Blaton, M.R., Eisenstein, D., Hogg, D. W., Schlegel, D. J., \& Brinkmann, J. 2005, ApJ, 629, 143 
Bond, J., R., Kofman, L., \& Pogosyan, D. 1996, Nature, 380, 603

Cervantes-Sodi, B. \& Hernandez, R. 2008, preprint

Choi, Y. Y., Park, C., \& Vogeley, M. 2007, ApJ, 658, 884

Colless et al. 2001, MNRAS, 328, 1039

Coziol, R., \& Plauchu-Frayn 2007, AJ, 133, 2630

Croton, D. J., Gao, L., \& White, S. D. M. 2007, MNRAS, 374, 1303

Dalal, N., White, M., Bond, J. R., \& Shirokov, A. 2008, preprint arXiv:0803.3453]

de Vaucouleurs, G., de Vaucouleurs, A., Corwin, H. G., Jr., Buta, R. J., Patuerl, G. \& Fouque, P. 1991, Third Reference Catalogue of Bright Galaxies (New York':Springer)

Desjacques, V. 2007, preprint (astro-ph/0707.4671)

Dressler, A. 1980, ApJ, 236, 351

Erdogdu, P. et al. 2006, MNRAS, 373, 45

Gao, L., Volker, S., \& White, S. D. M. 2005, MNRAS, 363, L66

Gomez, P. et al. 2003, ApJ, 584, 210

Goto, et al. 2003, MNRAS, 346, 601

Hernandez, R., \& Cervantes-Sodi, B. 2006, MNRAS, 368, 351

Hernandez, R. et al., 2007, AJ, 375, 163

Haynes, M. P. \& Giovanelli, R. 1984, AJ, 89, 758

Hockney, R. W. \& Eastwood, J. W. 1988, Computer Simulation Using Particles (New York: Taylor \& Francis)

Huchra, J. et al. 2005, in Nearby large scale structures and the zone of avoidance (ed. Fairall, F. \& Woudt, P. A.) 135-142 (ASP Conf. Ser. 329, Astronomical Society of the Pacific, Cape Town)

Kaiser, N. 1984, ApJ, 284, L9

Kuehn, F. \& Ryden, B. S. 2005, ApJ, 634, 1032 
Lauberts, A. 1982, ESO/Uppsala Survey of the ESO(B) Atlas. (Garching:ESO)

Lee, J. 2006, ApJ,

Lee, J. \& Erdogdu, P. 2007, ApJ,

Lewis, I., et al. 2002, MNRAS, 334, 673

Mateus, A., Sodre Jr, L. Cid Fernandes, R. \& Stasinska, G. 2007, MNRAS, 374, 1457

Mo, H. J., Mao, S. \& White, S. D. M. 1998, MNRAS, 295, 319

Navarro, J.F., Abadi, M.G., \& Steinmetz, M. 2004, ApJ, 613, L41

Nilson, P. 1974, Uppsala Astron. Obs. Ann., 6.

Owers, M. S. 2007, MNRAS, 381, 494

Pandey, B., \& Bharadwaj, S. 2006, MNRAS,

Pandey, B., \& Bharadwaj, S. 2008, MNRASin press

Park, C., Choi, Y. Y., Vogeley, M. S., Gott, J. R.,\& Blanton, M. R. 2007, ApJ, 658, 898

Park, C., Gott, J. R., \& Choi, Y. Y. 2008, ApJin press

Patiri, S. G., Cuesta, A. J., Prada, F., Betancort-Rijo, J., \& Klypin, A. 2006, ApJ, 652, 75

Postman, M., \& Geller, M. J., 1984, ApJ, 281, 95

Press, W. H., Teukoksky, S. A., Vetterling, W. T. \& Flannery, B. P. 1992, Numerical Recipes in FORTRAN (Cambridge : Cambridge Univ. Press)

Roediger, E. \& Hensler, G. 2005, A\&A, 433, 875

Rojas, R. R., Vogeley, M. S., Hoyle, F., \& Brinkmann, J. 2005, 624, 571

Sandvik, H., Moller, O., Lee, J.,\& White, S. D. M. 2007, MNRAS, 377, 234

Shen, J., Abel, T., Mo, H. J.,\& Sheth, R. K. 2006, ApJ, 645, 783

Sheth, R.,\& Tormen, G. 2006, MNRAS, 349, 1464

Springel, V. et al. 2005, Nature, 435, 629

Tanaka, M., Goto, T., Okamura, S., Shimasaku, K., \& Brinkman, J. 2004, AJ, 128, 2677 
Trujillo, I., Carretero, C., \& Patiri, S. 2006, ApJ, 610, L111

Vogeley, M. S. et al. 2004, Outskirts of Galaxy Clusters, Proceedings IAU Colloquium No. 195

van den Bosch, F. C., Aquino, D., Yang, X., Mo, H. J., Pasquali, A., Mcintosh, D. H., Weinmann, S. M., \& Kang, X. 2007, MNRAS, submitted (astro-ph/0710.3164)

West, M. J. 1989, ApJ, 347, 610

West, M. J. 1994, MNRAS, 268, 79

Whitmore, B. C., Gilmore, D. M., \& Jones, C. 1993, ApJ, 407, 489 
Table 1.

\begin{tabular}{cccc}
\hline \hline Subsample & Class & Type & $N_{g}$ \\
\hline E & Ellipticals & cE,E0,E0-1,E+ & 1008 \\
L & Lenticulars & $\mathrm{S} 0^{-}, \mathrm{S} 0^{\circ}, \mathrm{S} 0^{+}$ & 2532 \\
SI & Spirals & S0a,Sa & 1761 \\
SII & Spirals & Sab,Sb & 3175 \\
SIII & Spirals & Sbc,Sc & 4507 \\
SIV & Spirals & Scd,Sd,Sdm,Sm & 2679 \\
\hline
\end{tabular}




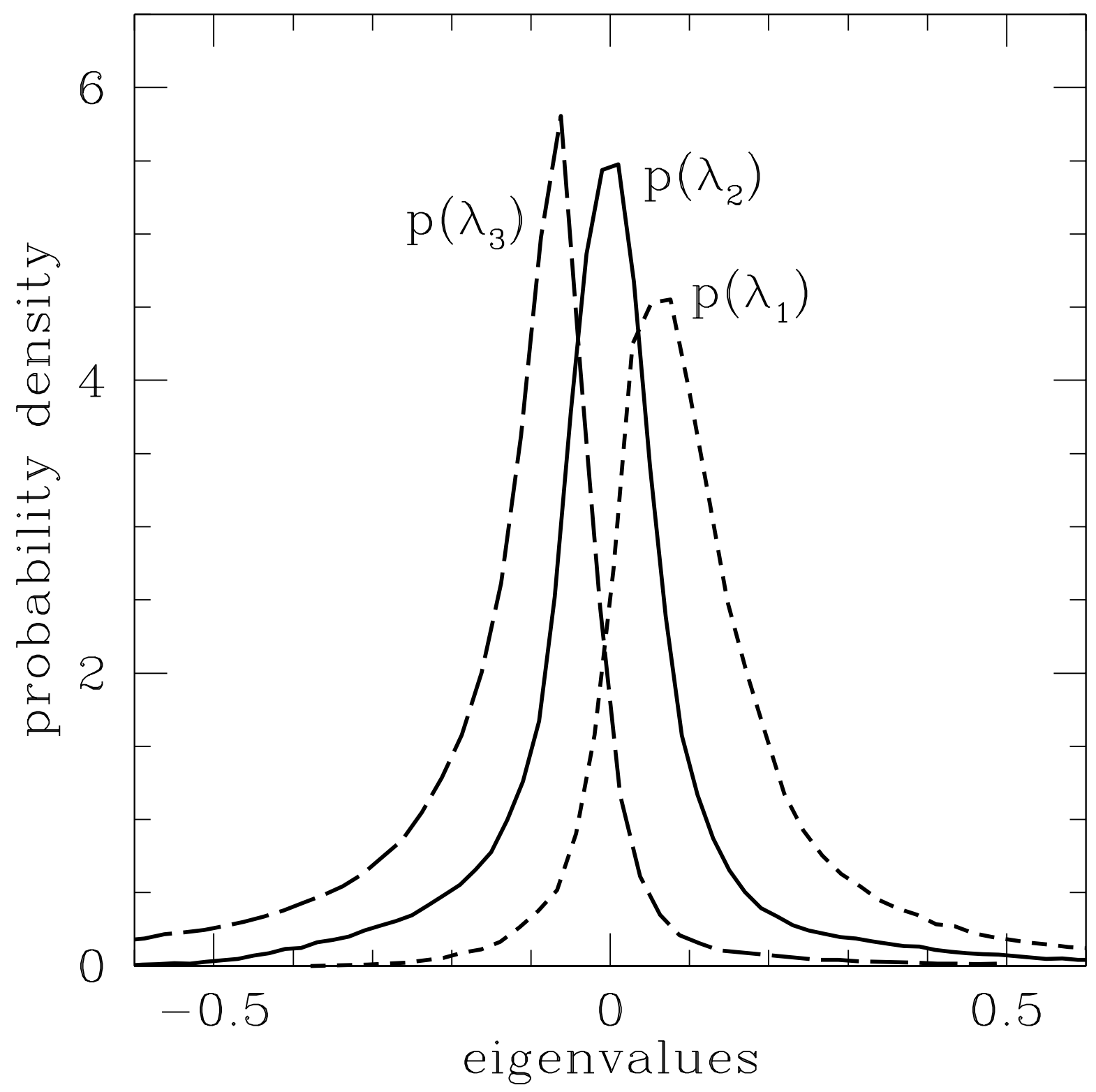

Fig. 1.- The probability density distributions of the three eigenvalues of the 2MRS tidal shear field. 

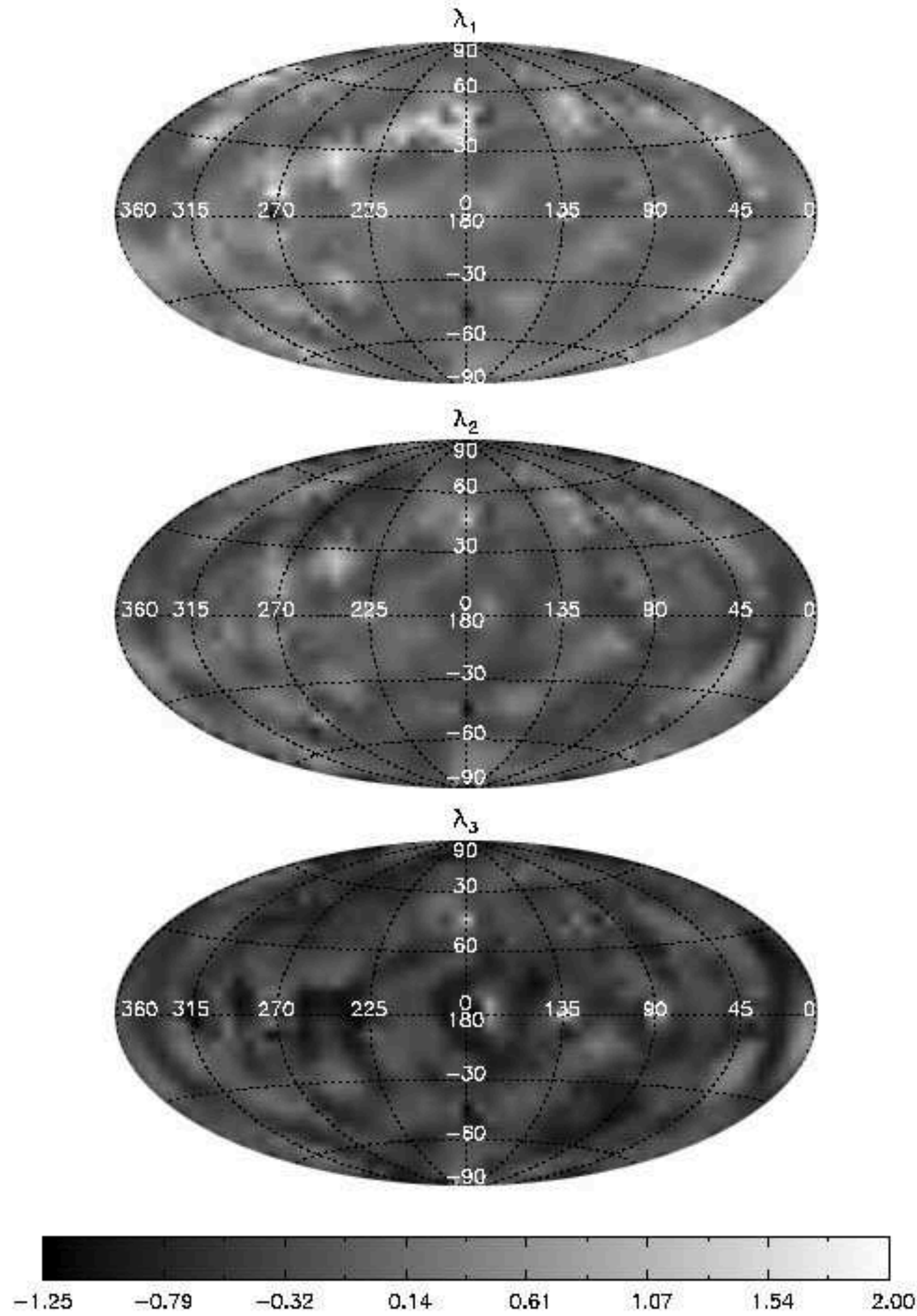

Fig. 2.- The Aitoff Projections of the tidal shear eigenvalue field in Supergalactic coordinates at $8000 \mathrm{~km} / \mathrm{s}: \lambda_{1}$ (top), $\lambda_{2}$ (middle), $\lambda_{3}$ (bottom) 


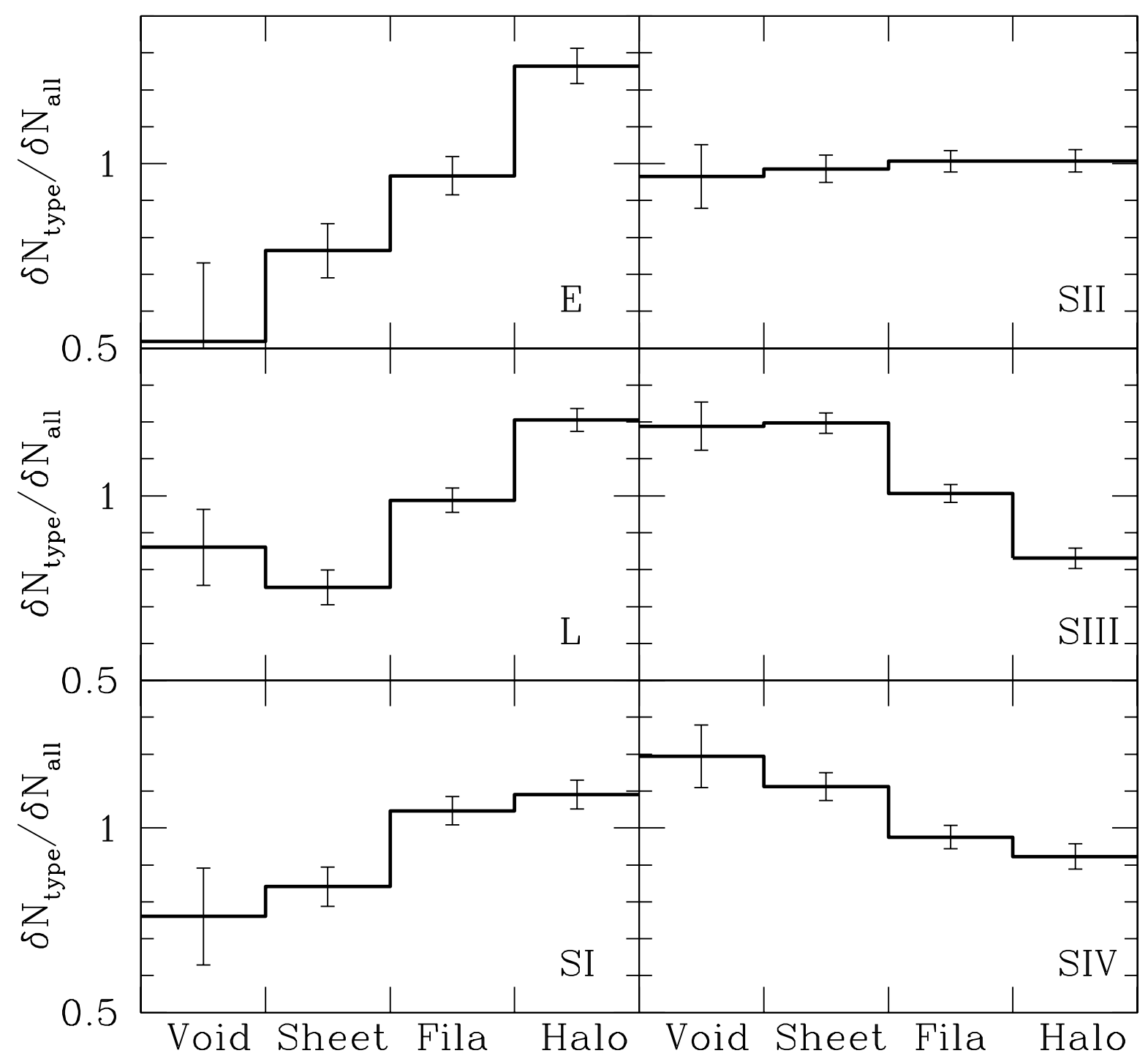

Fig. 3.- The conditional number densities of the galaxies located in the void-like, sheet-like, filament-like and halo-like regions for the six subsamples with Poisson errors. 


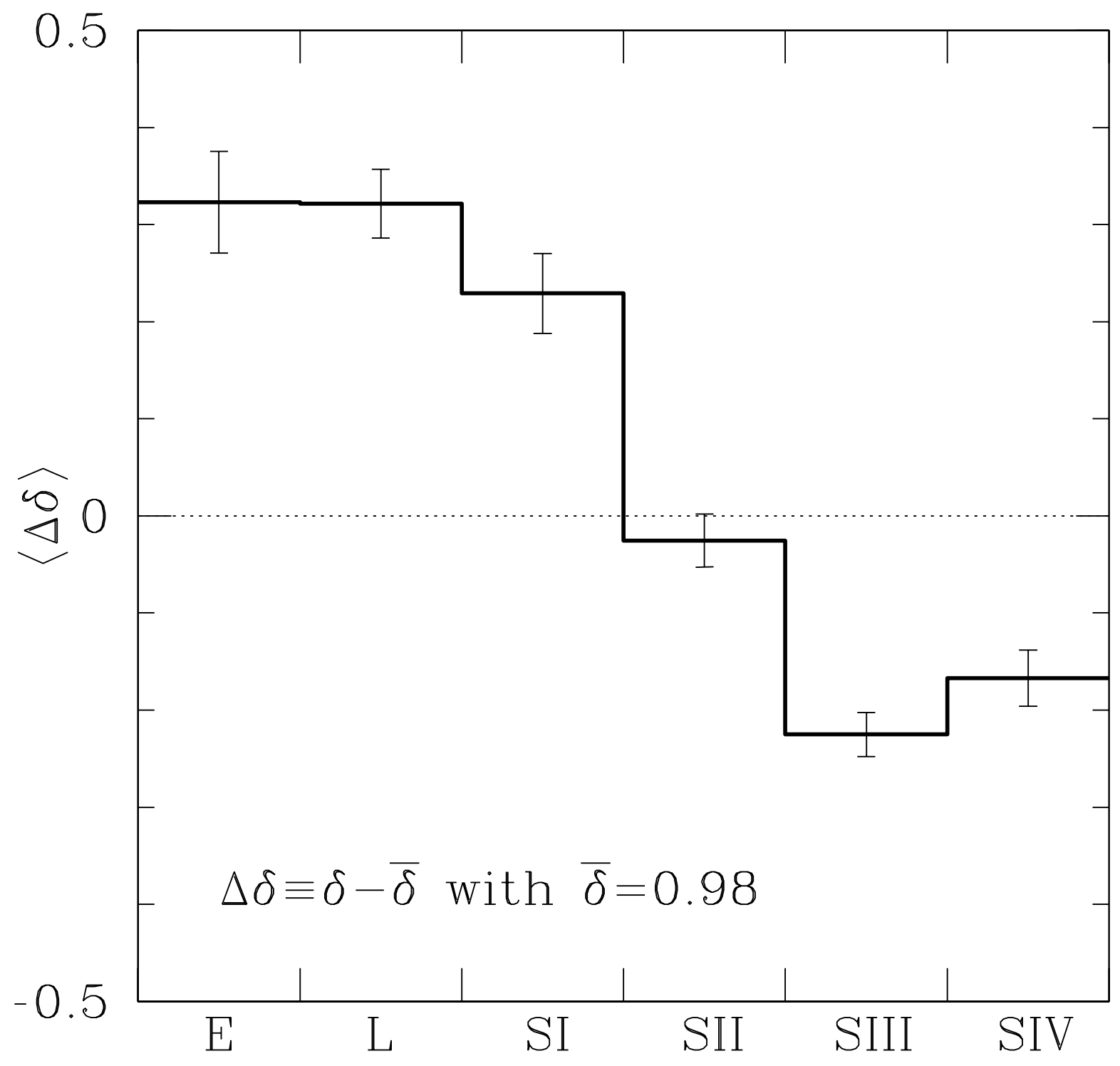

Fig. 4.- Mean of the density difference averaged over the regions where the Tully galaxies of each subsample are located. The Tully galaxies are classified into six subsamples according to the galaxy morphological type. The errors represent one standard deviation in the measurement of the mean values. 


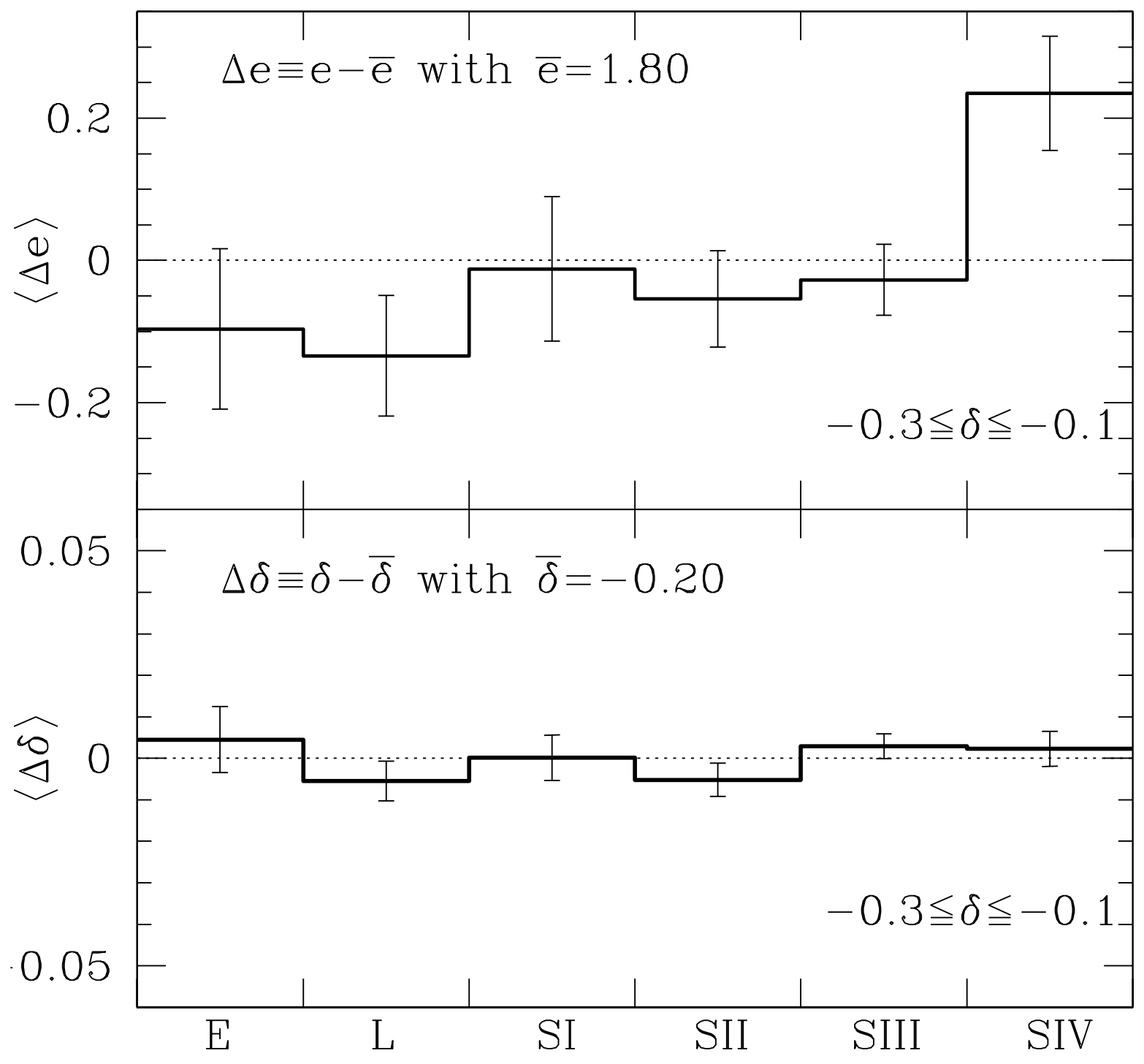

Fig. 5.- Mean of the ellipticity difference (top) and the density difference (bottom) averaged over each subsample with $\delta$ in the range of $[-0.3,-0.1]$. 


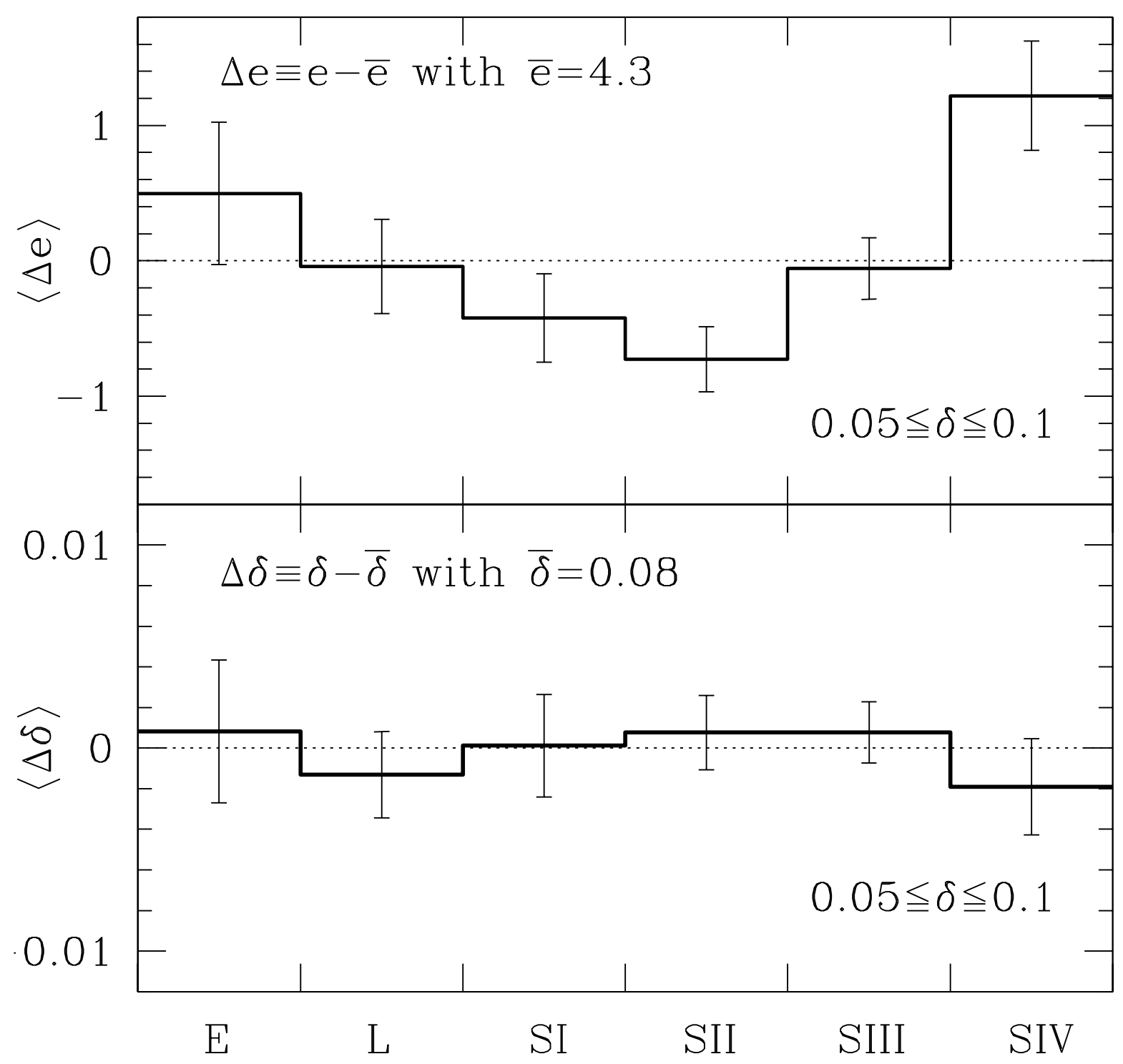

Fig. 6. - Same as Fig. 5 but with $\delta$ in the range of $[0.05,0.1]$. 


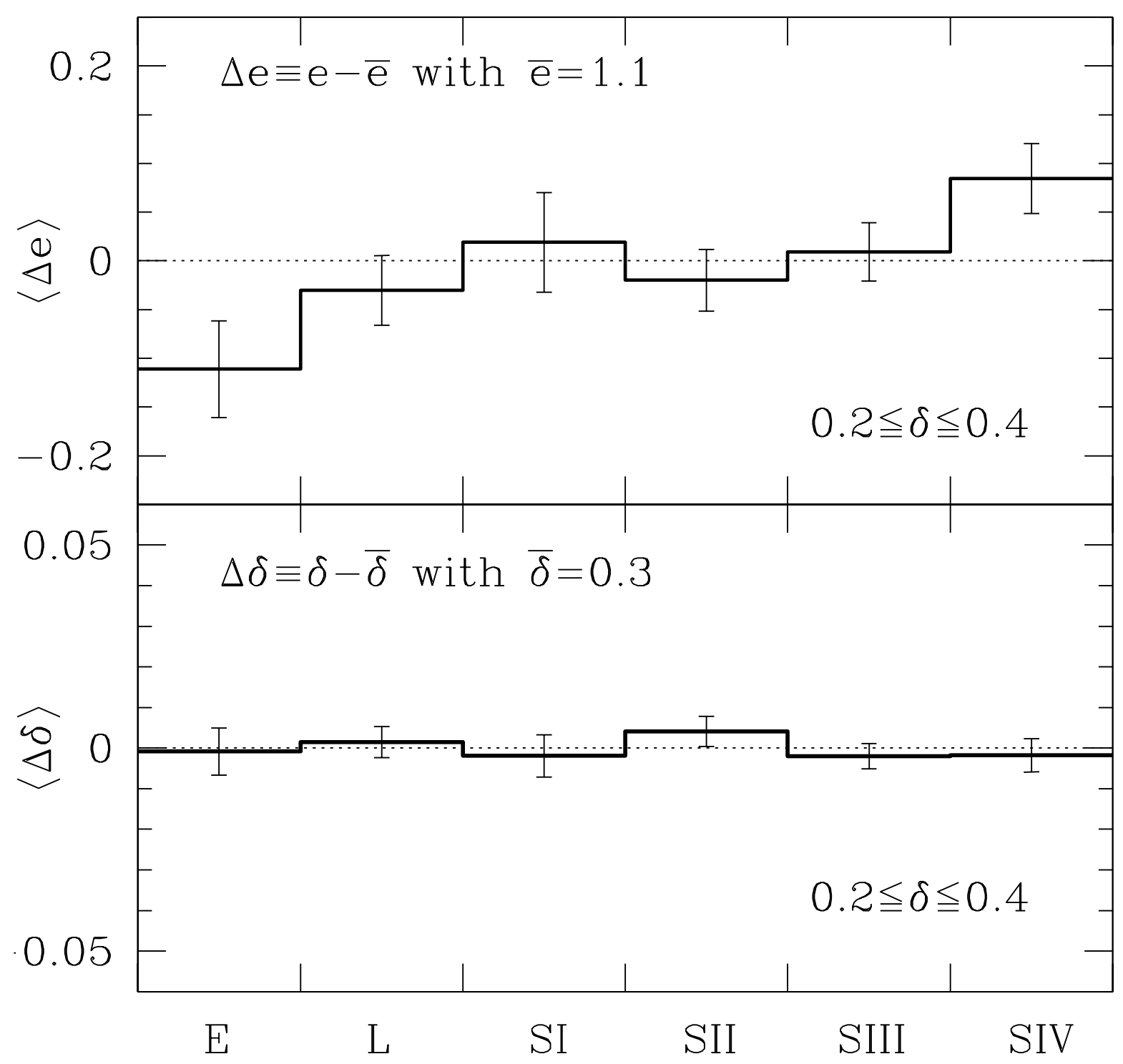

Fig. 7.- Same as Fig. 5 but with $\delta$ in the range of $[0.2,0.4]$. 


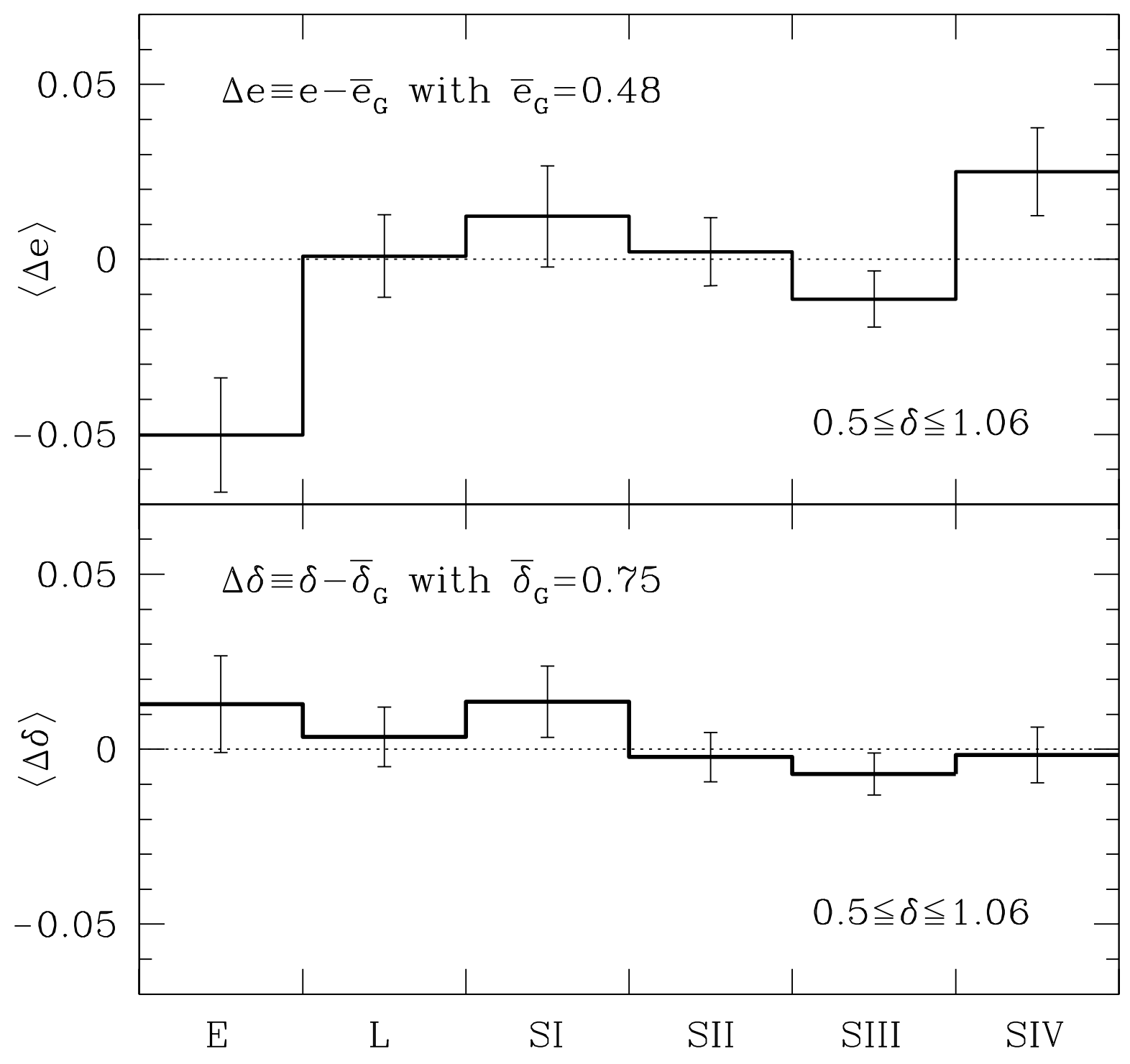

Fig. 8. - Same as Fig. 5 but with $\delta$ in the range of $[0.5,1.06]$. 